Sehbehinderung, Blindheit, Wirbelsäulen- und Gelenksdeformitäten mit Gehunfähigkeit gemildert und die Lebensqualität der Betroffenen deutlich verbessert werden. Die exakte Diagnose ist für die Eltern sehr wichtig, um in der genetischen Beratung und mit der nun möglichen Pränatal-
Diagnostik ein Bewusstsein für Familienplanung zu entwickeln.

Schlüsselwörter: Bilaterale kongenitale Katarakt, CCFDNSyndrom, Phänotypisierung, Genotypisierung.

\title{
Augenbefunde der Kinder der Blindenschule Peja/Kosovo
}

\section{H. Gruber}

Oberärztin am Zentralklinikum St. Pölten, Abteilung für Neuroophthalmologie, okuloplastische und Orbitachirurgie, Schulärztin des Bundes-Blindenerziehungsinstitutes Wien

Seit 2001 besteht eine Partnerschaft des Bundes-Blindenerziehungsinstitutes in Wien mit der Blindenschule in Peja im Kosovo.

Die augenärztliche Versorgung im Kosovo wäre nicht so schlecht, da sie aber privat bezahlt werden muss und sich viele Eltern die Augenarztbesuche nicht leisten können, gab es von den Schülern keine, sehr mangelhafte oder sehr alte Befunde. Die blindenpädagogischen Maßnahmen waren dadurch beeinträchtigt.

Im Rahmen eines neuerlichen Projektes habe ich zusammen mit Frau Claudia Lutterschmidt, diplomierte Orthoptistin am Zentralklinikum St. Pölten, 48 Kinder der Schule untersucht und die Befunde bezüglich Schwarzschrift- oder Braille-Beschulung mit dem Lehrpersonal besprochen.
Vergleicht man die Ursachen der Sehbehinderung oder Blindheit der kosovarischen mit den österreichischen Schülern und Schülerinnen, so fällt z. B. auf, dass in Wien ein großer Teil unserer Kinder eine Frühgeborenenretinopathie hat, in Peja waren es nur zwei. Wahrscheinlich überleben weniger frühgeborene Kinder und mehrfachbehinderte, sehgeschädigte Kinder kommen erst gar nicht in die Schule.

Einige Umstände haben uns sehr betroffen gemacht: 1. die schlechte Versorgung mit Hilfsmitteln - von der Brille bis zur Augenprothese, 2. die unbehandelten Sekundärglaukome, 3. die Tatsache, dass die Kinder nach Absolvierung ihrer Schulpflicht keine Arbeitsmöglichkeiten vorfinden.

Aus diesem Grund überlegen wir zusammen mit der Organisation Licht für die Welt - Christoffel-Blindenmission den Aufbau einer Blindenwerkstätte im Kosovo.

\section{Neuroophthalmologische Aspekte der Blepharoptosis: Teil 2: Differentialdiagnose}

\section{A. Ettl}

Abteilung für Neuroophthalmologie, okuloplastische und Orbitachirurgie, Zentralklinikum St. Pölten (Leiter: Prim. Univ.-Doz. Dr. Armin Ettl)

Problemstellung: Darstellung der Ursachen der Ptosis aus differentialdiagnostischer Sicht.

Methoden: Übersichtsvortrag mit Präsentation eigener Patienten. Nach Ausschluss einer Pseudoptosis (verschwindet nach Beseitigung der Ursache, beispielsweise einer Heberparese $=$ MED) führen uns Anamnese und Klinik zu Leitsymptomen und -befunden und in weiterer Folge aufgrund der präsentierten Tabellen zur Diagnose.

Ergebnisse: Beispiele differentialdiagnostischer Überlegungen: 1. Kongenitale Ptosis: Levatordystrophie, kong. Oculomotorius (III)-Parese, Horner-Syndrom, div. Myopathien, Myasthenia gravis (MG) und zahlreiche Syndrome wie Fibrosesyndrom (CFEOM). Vererbung, aber spätere Manifestation: Dystrophia myotonica, CPEO, Kearns-SayreSyndrom. 2. Akute Ptose: III-Parese bei Aneurysma, Karotisdissection etc. 3. Statische Ptosis: Levatordystrophie, strukt. def. Myopathien. 4. Intermittierende Ptosis: III-Parese mit zyklischen Spasmen, ophthalmoplegische Migräne, MG. 5. Transiente Ptosis: TIA. 6. Ermüdbarkeit: MG, Levatordehiszenz, Hirnstammtumor. 7. Beidseitige Ptosis: nucleäre III-Parese, CPEO, MG. 8. Unilaterale Ptosis: periphere IIIParese, MG. 9. Blickabhängige Ptosis: Levatordystrophie mit und ohne Synkinesien, aberrante Regeneration. 10. Kopfschmerz: z. B. Clusterkopfschmerz, Migräne, Tolosa-HuntSyndrom, Herpes Zoster, Arteriitis temporalis, Aneurysma, Karotisdissektion. 11. Rotes Auge: Clusterkopfschmerz, Karotis-Sinus cavernosus-Fistel. 12. Mydriasis: III-Parese. 13. Miosis: Horner-Syndrom. 14. Mittelweite Pupille: IIIParese + Horner-Syndrom bei Sinus-cavernosus-Syndrom. 15. Bilaterale, symmetrische Motilitätsstörung: Myopathien, Fibrosesyndrom (CFEOM). 16. Asymmetrische Motilitätsstörung mit Diplopie: III-Parese, MG. 17. Hebungseinschränkung: Doppelheberparese (MED), CFEOM, MG. 18. Marcus-Gunn-Synkinesie: Levatordystrophie, MED, CFEOM. 19. Exophthalmus: Orbitatumore, Myositis. 20. Sehverschlechterung: Orbitaspitzensyndrom, Mitochondriopathien, Nystagmus etc.

Schlussfolgerung: Ptosis ist ein Symptom, keine Erkrankung. Nach exakter Differentialdiagnose finden sich nicht selten als Ursache des herabhängenden Augenlides schwere neurologische Erkrankungen.

Schlüsselwörter: Ptosis, Neuroophthalmologie, Neurologie, Differentialdiagnose. 